\title{
ORFEUS echelle spectra: Molecular hydrogen in disk, IVC, and HVC gas in front of the LMC
}

\author{
H. Bluhm ${ }^{1}$, K. S. de Boer ${ }^{1}$, O. Marggraf ${ }^{1}$, and P. Richter ${ }^{1,2}$ \\ 1 Sternwarte, Universität Bonn, Auf dem Hügel 71, 53121 Bonn, Germany \\ 2 Washburn Observatory, University of Wisconsin-Madison, 475 N. Charter Street, Madison, WI 53706, USA \\ Received 5 September 2000 / Accepted 17 November 2000
}

\begin{abstract}
In front of the LMC molecular hydrogen is found in absorption near $0 \mathrm{~km} \mathrm{~s}^{-1}$, being local disk gas, near $+60 \mathrm{~km} \mathrm{~s}^{-1}$ in an intermediate velocity cloud, and near $+120 \mathrm{~km} \mathrm{~s}^{-1}$, being a high velocity halo cloud. The nature of the gas is discussed based on four ORFEUs far UV spectra of LMC stars and including data from the ground and from the IUE satellite. The local gas is cool and, given a span of sight lines of only $2.5^{\circ}$, rather fluffy. The fractional abundance of $\mathrm{H}_{2}$ varies from $\log f=\log \left[N\left(\mathrm{H}_{2}\right) /\left(2 \cdot N\left(\mathrm{H}_{2}\right)+N(\mathrm{H} \mathrm{I})\right)\right]=-5.4$ to -3.3 . Metal depletions (up to -1.7 dex for $\mathrm{Fe}$ ) are typical for galactic disk gas. In the intermediate and high velocity gas an apparent underabundance of neutral oxygen points to an ionization level of the gas of about $90 \% . \mathrm{H}_{2}$ is detected in intermediate and high velocity gas towards HD 269546. In the intermediate velocity gas we find an $\mathrm{H}_{2}$ column density of $\log (N) \simeq 15.6$. The $\mathrm{H}_{2}$ excitation indicates that the line of sight samples a cloud at a temperature below $150 \mathrm{~K}$. Column densities are too small to detect the higher UV pumped excitation levels. The high velocity $\mathrm{H}_{2}(\log (N) \simeq 15.6)$ is highly excited and probably exposed to a strong radiation field. Its excitation temperature exceeds $1000 \mathrm{~K}$. Due to the radial velocity difference between the halo gas and the Milky Way disk, the unattenuated disk radiation is available for $\mathrm{H}_{2}$ excitation in the halo. We do not find evidence for an intergalactic origin of this gas; a galactic as well as a Magellanic Cloud origin is possible.
\end{abstract}

Key words. ISM: abundances - ISM: molecules - Galaxy: structure - ultraviolet: ISM

\section{Gas in front of the LMC}

Shortly after the launch of the International Ultraviolet Explorer (IUE) satellite, gas absorbing at small and large radial velocity was found in spectra of stars in the Magellanic Clouds (Savage \& de Boer 1979). The absorption by Milky Way disk gas, of course near $0 \mathrm{~km} \mathrm{~s}^{-1}$, included species like Ci, OI, Si II, Fe II, MgII and the like. Gas in the Large Magellanic Cloud (LMC) and Small Magellanic Cloud (SMC) is present in absorption at the velocity of each of these galaxies.

In the direction of the LMC gas was found also at intermediate velocities, near $+60 \mathrm{~km} \mathrm{~s}^{-1}$ and near $+120 \mathrm{~km} \mathrm{~s}^{-1}$ (Savage \& de Boer 1979). Both velocities were seen in absorption by the normal neutral species. Absorption by highly ionized species such as Si IV and C IV was present at non zero velocities as well. Clearly, an intermediate velocity cloud (IVC) and a high velocity cloud (HVC) had been detected.

Since then numerous further observations in the visual and the ultraviolet of other Magellanic Cloud stars confirmed these interstellar absorptions. In addition, probing at $21 \mathrm{~cm}$ outside the face of the LMC showed that in

Send offprint requests to: $\mathrm{H}$. Bluhm, e-mail: hbluhm@astro.uni-bonn.de particular the gas at $+120 \mathrm{~km} \mathrm{~s}^{-1}$ has a rather wide angular spread (de Boer et al. 1990). The latter observations gave strong support for the claim that the $+120 \mathrm{~km} \mathrm{~s}^{-1}$ gas is part of the galactic halo.

A new wavelength domain was opened for the observation of stars in the Magellanic Clouds with the ORFEUS space shuttle mission. The echelle spectrograph on board ORFEUS produces spectra between 900 and $1400 \AA$ with a spectral resolution of $\simeq 30 \mathrm{~km} \mathrm{~s}^{-1}$ (see Barnstedt et al. 1999). Thus interstellar absorption lines of molecular hydrogen $\mathrm{H}_{2}$ (and also of $\mathrm{O} \mathrm{VI}$ ) became accessible.

Analyses of the LMC part of the ORFEUs absorption spectra have resulted in the detection of $\mathrm{H}_{2}$ (de Boer et al. 1998) and in a study of the $\mathrm{H}_{2} / \mathrm{CO}$ ratio (Richter et al. 1999b). A full analysis of ORFEUS $\mathrm{H}_{2}$ observations in both Magellanic Clouds is presented by Richter (2000a).

Here we will analyse the absorption by $\mathrm{H}_{2}$ and metals in the neutral gas in the foreground of the LMC using ORFEus spectra (see Table 1). The data were obtained during the mission of Nov./Dec. 1996.

Detailed inspection of the ORFEUS LMC star spectra showed the existence of absorption by $\mathrm{H}_{2}$ at intermediate velocities, towards HD 269546. This is the third IVC seen in $\mathrm{H}_{2}$. The first IVC with $\mathrm{H}_{2}$ was found towards HD 93521 by Gringel et al. (2000), the second towards 
PG $0804+761$ by Richter et al. (2000b). The discovery of $\mathrm{H}_{2}$ in the $+120 \mathrm{kms}^{-1} \mathrm{HVC}$ was presented by Richter et al. (1999a).

\section{Data and data reduction}

The data were preanalysed by the oRfeus team in Tübingen and provided in the form of spectra order by order (Barnstedt et al. 1999). The interstellar absorption lines exhibit in part terrible blending, due to the rather densely packed absorptions by $\mathrm{H}_{2}$ together with the large $\left(\simeq 300 \mathrm{~km} \mathrm{~s}^{-1}\right)$ range in velocity covered by each absorption. Given the considerable blending, not each velocity component could be identified for all transitions individually. Only blend free lines and blend free portions of absorption profiles have been selected for the present study. The tabular material presented may therefore give the impression of inhomogeneity.

The target data are listed in Table 1. Examples of absorption profiles, both for $\mathrm{H}_{2}$ as well as metal lines, are given in Fig. 1 for each of the stars.

Spectra had also been obtained with the IUE satellite, except for LH 10:3120. We have included these spectra (from the public archive) in our analysis. Note that the IUE spectrum SWP 13347 is not of HD 269546 (here the IUE archive is at fault; see Chu et al. 1994). A preliminary analysis of the velocity and Si II column density in the IVC and HVC in front of the LMC based on IUE spectra of many LMC stars has been presented by Wierig \& de Boer (1998).

The intercomparison of ORFEUS and IUE data in the spectral range common to both instruments shows that the absorption profiles agree well. We have therefore combined the data from ORFEUs with those available from the IUE and can so obtain a more complete picture for the nature of the gas in the three velocity components studied.

Data of $\mathrm{H}$ I emission at $21 \mathrm{~cm}$ are available from the literature (McGee et al. 1983; McGee \& Newton 1986; de Boer et al. 1990).

At this point we have to dwell briefly on the velocity scale used for the data. The zero point of the velocity scale of the ORFEUS spectra is heliocentric for stars whose position in the sky was perfectly known and which were observed at the centre of the ORFEUs aperture (Barnstedt et al. 1999). Neither of the requirements must have been fulfilled for our stars. A similar situtation holds for the IUE data. In the four analysed ORFEUs spectra the zero points of the velocity scales should be correct within $10 \mathrm{~km} \mathrm{~s}^{-1}$.

\section{Toward column densities and gas properties}

Each velocity component was analysed in the following manner:

The equivalent widths were measured by trapezium or Gaussian fits. Their uncertainties are calculated from the estimated error in the choice of the continuum and the photon statistics, which were taken into consideration using a formula by Jenkins et al. (1973).
Table 1. LMC stars observed with the ORFEus echelle spectrograph

\begin{tabular}{|c|c|c|c|c|c|}
\hline Star & $\begin{array}{l}l \quad b \\
{[\text { degree] }}\end{array}$ & $\begin{array}{c}V \\
{[\mathrm{mag}]}\end{array}$ & Sp.T. & $E(B-V)$ & $\begin{array}{c}\text { exp.time } \\
{[\mathrm{s}]}\end{array}$ \\
\hline LH 10:3120 & $277.2-36.1$ & 12.80 & $\mathrm{O} 5.5 \mathrm{~V}$ & 0.17 & 6528 \\
\hline Sk -67166 & $277.8-32.5$ & 12.27 & O5e & 0.09 & 6188 \\
\hline HD 36402 & $277.8-33.0$ & 11.50 & $\mathrm{OB}+\mathrm{WC} 5$ & $5 \leq 0.02$ & 6764 \\
\hline HD 269546 & $279.3-32.8$ & 11.30 & $\mathrm{~B} 3+\mathrm{WN} 3$ & $\leq 0.02$ & 6388 \\
\hline
\end{tabular}

References for $E(B-V)$ values: LH 10:3120: Parker et al. (1992), Sk -67 166: Wilcots et al. (1996), HD 36402: de Boer \& Nash (1982), HD 269546: Vacca \& Torres-Dodgen (1990). We use the names by which the LMC stars are best known. Other names are: $\mathrm{Sk}-67166=\mathrm{HD} 269698, \mathrm{HD} 36402=\mathrm{Sk}-67104$, HD $269546=\mathrm{Sk}-6882$.

Table 2. Analysed metal absorption lines. Measurements in ORFEUS and IUE spectra are denoted by $o$ and $i$, respectively

\begin{tabular}{lllll}
\hline Ion & \multicolumn{4}{l}{ Transition $($ wavelength in $\AA$ ) } \\
\hline Si II & $1190 i, o$ & $1193 i, o$ & $1260 i, o$ & $1304 i, o$ \\
& $1526 i$ & $1808 i$ & & \\
Fe II & $1125 o$ & $1142 o$ & $1144 o$ & $2344 i$ \\
& $2374 i$ & $2382 i$ & $2586 i$ & $2600 i$ \\
S II & $1250 i, o$ & $1253 i, o$ & $1259 i, o$ & \\
O I & $936 o$ & $976 o$ & $1039 o$ & $1302 i, o$ \\
Mg I & $2852 i$ & & & \\
Mg II & $2796 i$ & $2803 i$ & & \\
\hline
\end{tabular}

For $\mathrm{H}_{2}$ we constructed (using $f$-values from Morton \& Dinerstein 1976) the curve of growth for each $J$-level independently. These curves generally have the same $b$ value. Column densities then follow, also for those levels for which possibly only one line could be measured.

The various rotational levels of $\mathrm{H}_{2}$ can be excited through collisions with other particles, leading mostly to population of the lower $J$ levels, and through photons, leading mostly to population of the higher $J$ levels (Spitzer \& Zweibel 1974). The latter process is also called UV pumping. We have fitted a Boltzmann distribution to the column densities derived for the rotational states. This results, for the low $J$ levels, in a value for the kinetic temperature of the gas. For the high $J$ levels the fit produces a value merely indicating the level of UV pumping. The nature of the high level excitation can, in principle, be used to get information about the FUV radiation density (Wright \& Morton 1979).

For each atomic species ( $f$-values from the compilation by Morton 1991 and from the review by Savage \& Sembach 1996) a curve of growth was constructed, too. The total $b$-value is mostly based on the curve of growth of Si II and Fe II. Column densities were derived in the usual manner. For those species having too few absorption lines the column density was derived by shifting the data to the adopted curve of growth. 

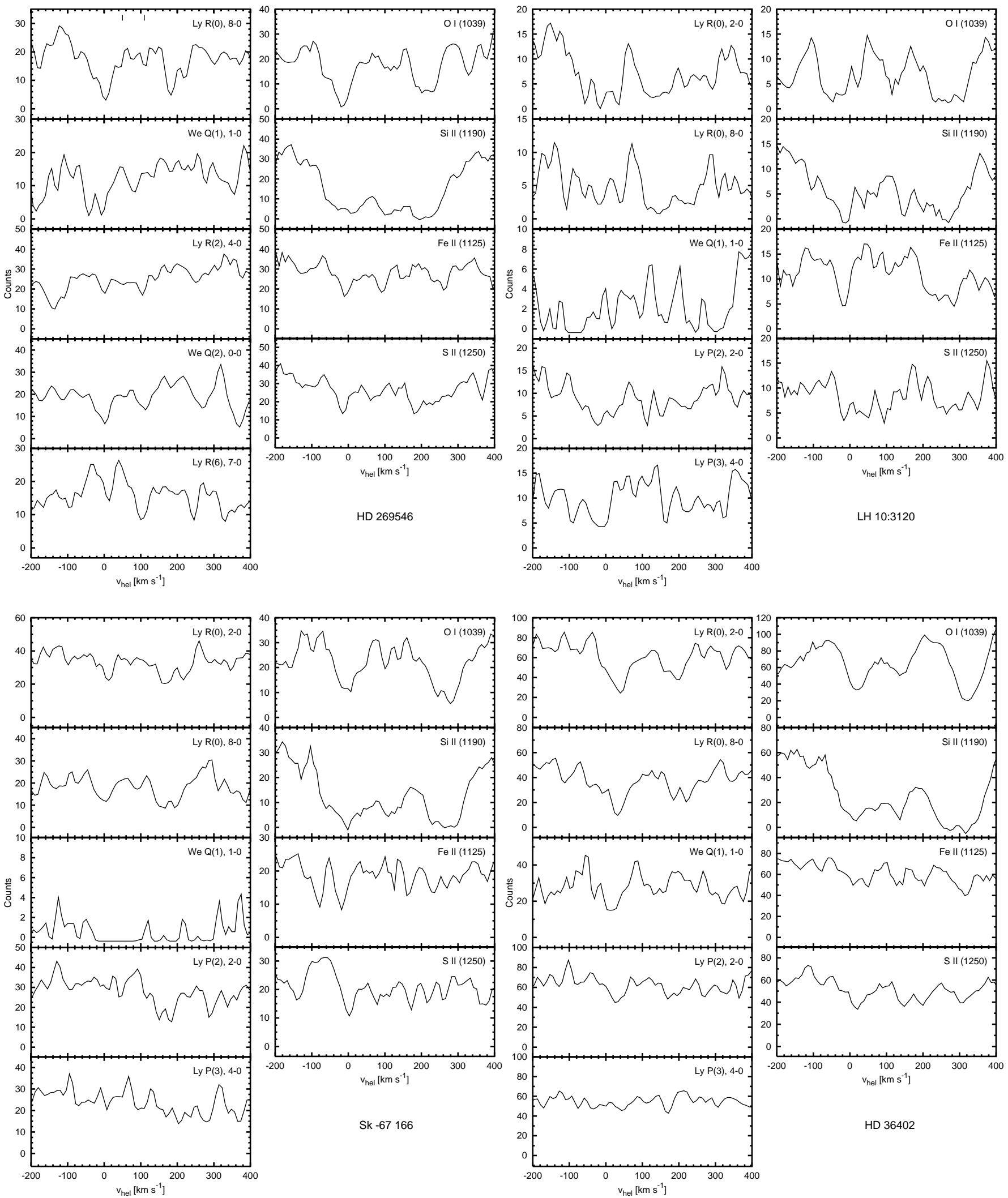

Fig. 1. For each line of sight a sample of five $\mathrm{H}_{2}$ and four metal lines as measured with ORFEus is shown. Additional transitions within the range of the plots are: Ly $\mathrm{R}(1)$, 8-0, Ly P(4), 9-0 [Ly $\mathrm{R}(0)$, 8-0]; Ly P(5), 11-0, Ly R(3), 10-0), We R(3), 1-0, We $\mathrm{R}(0)$, 1-0, We R(1), 1-0 [We Q(1), 1-0]; Ly P(1), 4-0 [Ly R(2), 4-0]; We R(4), 0-0, We R(3), 0-0 [We Q(2), 0-0]; Ly P(3), 6-0, Cl I [Ly R(6), 7-0]; Fe II, Ly R(3), 2-0 [Ly P(2), 2-0]; Ly R(2), 5-0 [O I]; S III [Si II $1190 \AA$ ]; P II [Fe II $1125 \AA$ ] 
The given errors of the column densities are based on the errors of the equivalent widths and the estimated uncertainty of the $b$-value.

For LH 10:3120 no IUE spectrum is available and the ORFEUS spectrum has a relatively low countrate (mostly between 10 and 20 counts). For that reason only a few strong lines could be measured.

\section{Densities and temperature in the clouds}

Above we have given a general description of the methods of analysis employed to derive velocities, column densities, temperatures, excitation conditions, etc. from the data. Here we will discuss the results for each velocity component as derived from the available lines of sight. $\mathrm{H}_{2}$ equivalent widths and column densities are listed in Tables 3-6. Metal and H I column densities are given in Table 7.

\subsection{Disk gas}

Gas near $0 \mathrm{kms}^{-1}$ is gas in the local Milky Way disk, whose thickness is normally indicated by a scale height of $\simeq 100$ pc. However, gas is known to exist slightly further out, at distances up to $1 \mathrm{kpc}$ (Lockman et al. 1986). The four ORFEUs lines of sight span about $3^{\circ}$ on the sky which, at a distance of less than $1 \mathrm{kpc}$, represents a lateral extent of less than $60 \mathrm{pc}$.

The column densities of metal species do not show significant variations between the different lines of sight, except perhaps for Fe II (see Table 7). For oxygen we have to note that the absorption lines in the ORFEUs spectra are contaminated by geocoronal emission. The absorption profile is partly filled in by the emission so that the measured equivalent widths of the disk component are lower limits, while the IVC and HVC lines are not affected. This explains why the O I disk depletions are given as lower limits in Table 8.

The absorption by the disk gas probably consists of at least two unresolved components. This is supported by the high Doppler-parameters ( $b$ between 15 and $22 \mathrm{~km} \mathrm{~s}^{-1}$ ) of the metal curves of growth. A clear deviation from the single cloud curve is visible in the rise in the level of the flat part of the curve of growth of the Sk -67166 disk component. The $\mathrm{H}_{2}$ measurements support the suspicion of the presence of more than one component, since for this sight-line the radial velocity of $\mathrm{H}_{2}$ lies asymmetric within the velocity width of the metal lines. Towards HD 36402 two $\mathrm{H}_{2}$ absorption components ( $\mathrm{A}$ and $\mathrm{B}$ ) on either side of the mean metal radial velocity are detected. The spectra of LH 10:3120 are too noisy to recognize the double nature of the absorption. Optical Ca II spectra at higher resolution (as shown e.g. in Wayte 1990) towards other LMC stars confirm the existence of velocity structure in disk gas in this direction.

The metals are moderately depleted in the disk component, e.g. $\log (\mathrm{S} / \mathrm{H})-\log (\mathrm{S} / \mathrm{H})_{\odot}=-0.5$ to -0.8 and $\log (\mathrm{Fe} / \mathrm{H})-\log (\mathrm{Fe} / \mathrm{H})_{\odot}=-1.0$ to -1.7 . This points to

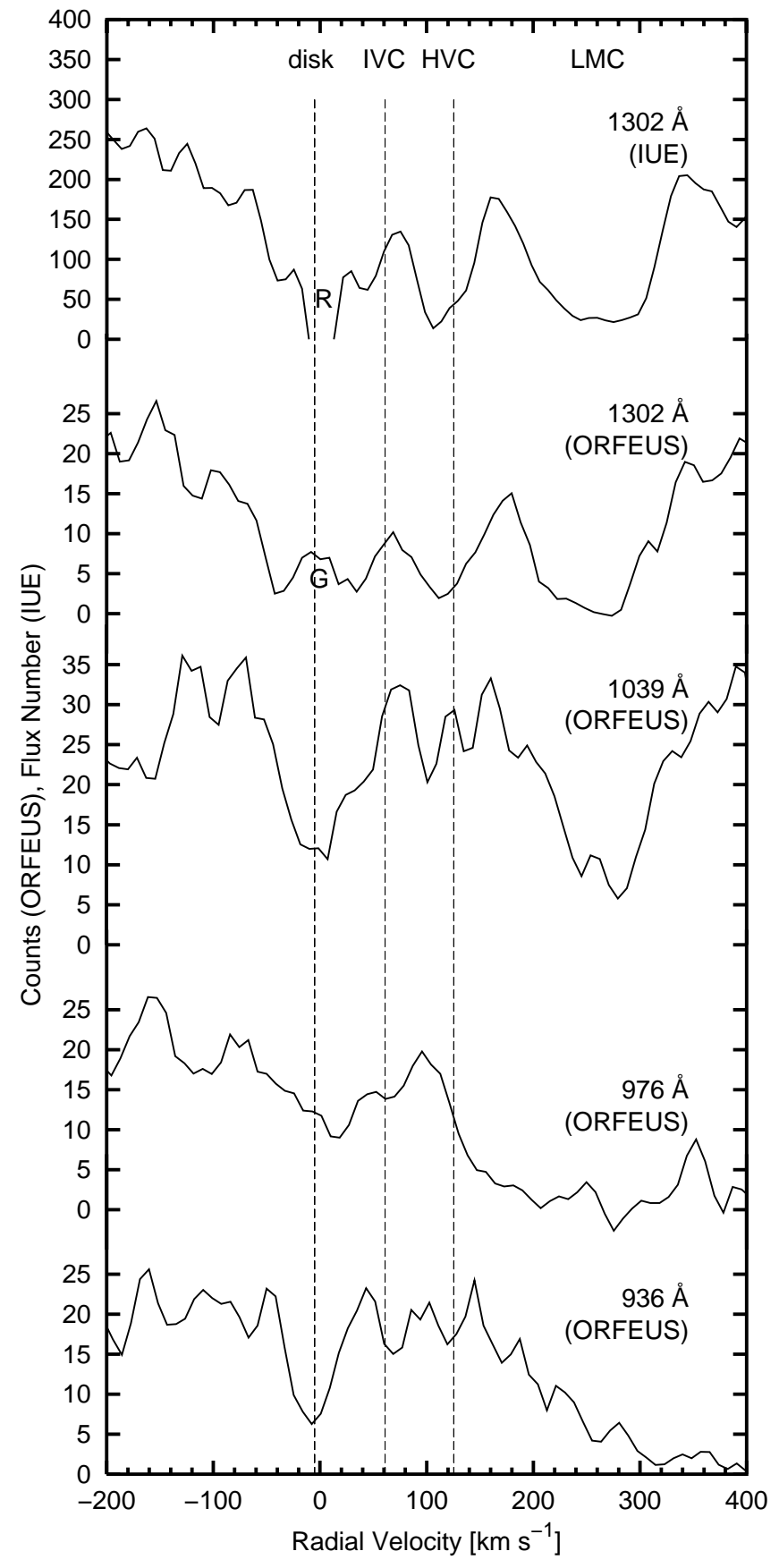

Fig. 2. Profiles of some O I lines in the spectrum of Sk -67166 . The radial velocity is heliocentric and not corrected for possible pointing errors. Note the reseaux $(R)$ at the centre of the $1302 \AA$ line in the IUE spectrum and the geocoronal emission $(\mathrm{G})$ at the same place in the corresponding ORFEus spectrum. Significant contamination of other O I lines by airglow is not as obvious, but cannot be excluded

the existence of dust on these lines of sight, but not in a large quantity.

The $\mathrm{H}_{2}$ column densities for the local gas (see Table 5) scatter more than the $\mathrm{HI}$ and metal column densities. However, this is not surprising. $\mathrm{H}_{2}$ is expected to exist mainly in the cores of interstellar clouds, surrounded by 
Table 3. $\mathrm{H}_{2}$ equivalent widths of galactic disk gas in the spectra of LMC stars

\begin{tabular}{|c|c|c|c|c|c|c|}
\hline & \multirow[t]{2}{*}{$\lambda[\AA]$} & \multicolumn{5}{|c|}{$W_{\lambda}[\mathrm{m} \AA]$} \\
\hline & & HD 269546 & LH 10:3120 & $\mathrm{Sk}-67166$ & HD $36402(\mathrm{~A})$ & HD 36402 (B) \\
\hline Ly $R(0), 0-0$ & 1108.128 & $\mathrm{n}$ & $48 \pm 24$ & $<15$ & $\mathrm{n}$ & $49 \pm 14$ \\
\hline Ly $R(0), 2-0$ & 1077.138 & $121 \pm 19$ & $114 \pm 24$ & $28 \pm 8$ & $26 \pm 13$ & $87 \pm 17$ \\
\hline Ly $R(0), 4-0$ & 1049.366 & $\mathrm{~b} ?, \mathrm{n}$ & $\mathrm{b} ?, \mathrm{n}$ & $\mathrm{b} ?, \mathrm{n}$ & $28 \pm 8$ & $\mathrm{~b} ?, \mathrm{n}$ \\
\hline Ly $R(0), 8-0$ & 1001.826 & $104 \pm 19$ & $94 \pm 33$ & $\mathrm{n}$ & $26 \pm 12$ & $102 \pm 17$ \\
\hline Ly $R(0), 10-0$ & 981.441 & $\mathrm{n}, \mathrm{b} ?$ & $143 \pm 28$ & $\mathrm{n}$ & $\mathrm{n}$ & $\mathrm{n}$ \\
\hline Ly $R(0), 12-0$ & 962.978 & $\mathrm{~b} ?$ & f & $31 \pm 15$ & $23 \pm 12$ & $\mathrm{~b} ?, \mathrm{n}$ \\
\hline Ly $R(1), 0-0$ & 1108.634 & $58 \pm 18$ & $\mathrm{n}$ & $<16$ & $<13$ & $32 \pm 14$ \\
\hline We $Q(1), 0-0$ & 1009.772 & $96 \pm 18$ & $\mathrm{n}$ & $34 \pm 14$ & $25 \pm 7$ & $51 \pm 12$ \\
\hline Ly R(1), 1-0 & 1092.732 & b (LR0, 1-0, HVC) & $\mathrm{n} ?$ & $15 \pm 9$ & $\mathrm{n}$ & - \\
\hline We $Q(1), 1-0$ & 986.798 & $87 \pm 20$ & $49 \pm 29$ & f & $34 \pm 8$ & $50 \pm 9$ \\
\hline Ly R(1), 2-0 & 1077.698 & $60 \pm 18$ & $\mathrm{n}$ & $25 \pm 9$ & $19 \pm 9$ & - \\
\hline We $Q(1), 3-0$ & 947.425 & $\mathrm{~b} ?$ & $\mathrm{f}$ & $\mathrm{b} ?$ & $33 \pm 14$ & $\mathrm{~b} ?$ \\
\hline Ly R(1), 4-0 & 1049.958 & $97 \pm 18$ & $\mathrm{f}$ & $54 \pm 11$ & $21 \pm 10$ & $72 \pm 12$ \\
\hline Ly $R(1), 7-0$ & 1013.434 & $85 \pm 24$ & $98 \pm 29$ & $\mathrm{n}$ & $\mathrm{n}$ & \\
\hline Ly $R(1), 8-0$ & 1002.457 & $\mathrm{n}$ & $75 \pm 29$ & $40 \pm 11$ & $35 \pm 13$ & $56 \pm 14$ \\
\hline Ly $R(1), 12-0$ & 963.609 & $\mathrm{f}$ & $82 \pm 23$ & - & $\mathrm{n}$ & f \\
\hline Ly $R(1), 13-0$ & 955.067 & $\mathrm{f}$ & b (LR0) & $<32$ & $31 \pm 12$ & $65 \pm 18$ \\
\hline Ly $\mathrm{P}(2), 0-0$ & 1112.495 & b (FeII, CI) & $\mathrm{b}$ & $<32$ & $\mathrm{~b}$ & $\mathrm{n}$ \\
\hline Ly $\mathrm{P}(2), 1-0$ & 1096.439 & $\mathrm{n}$ & $69 \pm 25$ & $\mathrm{n}$ & $\mathrm{n}$ & $<15$ \\
\hline Ly $\mathrm{P}(2), 2-0$ & 1081.265 & $40 \pm 14$ & $76 \pm 24$ & $14 \pm 11$ & $<11$ & $18 \pm 9$ \\
\hline Ly $\mathrm{P}(2), 6-0$ & 1028.103 & $47 \pm 18$ & $\mathrm{f}$ & $36 \pm 22$ & $\mathrm{f}$ & $\mathrm{f}$ \\
\hline Ly $\mathrm{P}(2), 7-0$ & 1016.472 & $61 \pm 16$ & $42 \pm 22$ & $\mathrm{n}$ & $17 \pm 8$ & $16 \pm 9$ \\
\hline Ly $\mathrm{P}(2), 8-0$ & 1005.397 & $33 \pm 19$ & $56 \pm 23$ & $\mathrm{n}$ & $\mathrm{n}$ & $\mathrm{b}$ \\
\hline Ly $\mathrm{P}(3), 1-0$ & 1099.788 & $26 \pm 12$ & - & $17 \pm 12$ & $\mathrm{n}$ & $<32$ \\
\hline Ly $\mathrm{P}(3), 2-0$ & 1084.559 & $\mathrm{~b}$ & $39 \pm 28$ & $\mathrm{n}$ & $\mathrm{n}$ & $\mathrm{b}$ \\
\hline We $\mathrm{P}(3), 2-0$ & 970.560 & $\mathrm{n}, \mathrm{f}$ & $44 \pm 28$ & $\mathrm{n}$ & $\mathrm{f}$ & $\mathrm{f}$ \\
\hline Ly $\mathrm{P}(3), 4-0$ & 1056.469 & $50 \pm 16$ & - & $23 \pm 14$ & $<22$ & $13 \pm 6$ \\
\hline Ly $\mathrm{P}(3), 5-0$ & 1043.498 & $\mathrm{~b}$ & $\mathrm{~b}$ & $\mathrm{~b}$ & $\mathrm{~b}$ & $20 \pm 8$ \\
\hline Ly $\mathrm{P}(3), 15-0$ & 944.331 & $\mathrm{n}$ & $41 \pm 24$ & $23 \pm 21$ & $<20$ & $\mathrm{n}$ \\
\hline Ly $\mathrm{P}(4), 0-0$ & 1120.247 & $<20$ & $\mathrm{n}$ & - & - & $12 \pm 7$ \\
\hline Ly $\mathrm{P}(4), 2-0$ & 1088.794 & $27 \pm 13$ & $34 \pm 20$ & - & - & $\mathrm{n}$ \\
\hline Ly $\mathrm{P}(4), 3-0$ & 1074.313 & $\mathrm{n}$ & $22 \pm 17$ & - & - & $\mathrm{n}$ \\
\hline Ly $P(4), 5-0$ & 1047.554 & $44 \pm 15$ & $\mathrm{n}$ & $<18$ & - & $\mathrm{n}$ \\
\hline Ly $\mathrm{P}(5), 1-0$ & 1109.313 & $\mathrm{n}$ & - & $<21$ & - & $<16$ \\
\hline We $\mathrm{P}(5), 1-0$ & 997.640 & $\mathrm{n}$ & $<26$ & - & - & - \\
\hline Ly $\mathrm{P}(5), 8-0$ & 1017.009 & $35 \pm 14$ & - & - & - & - \\
\hline Ly $\mathrm{P}(5), 14-0$ & 960.265 & $<26$ & - & - & - & - \\
\hline
\end{tabular}

Wavelengths are taken from Morton \& Dinerstein (1976). Some equivalent widths have not been measured due to: (n) noise, (f) low flux, (b) blend. Some blends could not be identified.

the more extended atomic gas, and our lines of sight surely sample different parts of the molecular clouds.

The $\mathrm{H}_{2}$ Boltzmann excitation temperatures were determined for the lower levels $J=0$ and 1 as well as for 0 and 2. While the $J=0,1$ comparison is directly linked to the ortho- $\mathrm{H}_{2}$ to para- $\mathrm{H}_{2}$ ratio (OPR), which might not be fully thermalized, the $J=0,2$ comparison refers to the two lowermost para-states and thus gives the real kinetic temperature (at least as long as collisional excitation predominates in both states).
To investigate the upper UV pumping excited levels, we use the equivalent temperatures for the para-states 2 and 4 and for the ortho-states 3 and 5 , respectively. The temperatures are listed in Table 5, for some of the values only limits can be given.

For all lower states the Boltzmann excitation temperatures are found within a range of about 50 to $100 \mathrm{~K}$, as also found by Savage et al. (1977) for general galactic gas from Copernicus data. For HD 269546, LH 10:3120 and for component $\mathrm{B}$ of HD $36402 T_{01}$ and $T_{02}$ are 
Table 4. Halo $\mathrm{H}_{2}$ equivalent widths towards the LMC stars

\begin{tabular}{|c|c|c|c|c|c|c|c|c|c|}
\hline & \multirow[t]{3}{*}{$\lambda[\AA]$} & \multicolumn{8}{|c|}{$W_{\lambda}[\mathrm{m} \AA]$} \\
\hline & & \multirow{2}{*}{$\begin{array}{l}\text { HD } 269546 \\
\text { IVC }\end{array}$} & \multirow[b]{2}{*}{$\mathrm{HVC}$} & \multicolumn{2}{|c|}{ LH 10:3120 } & \multicolumn{2}{|c|}{$\mathrm{Sk}-67166$} & \multicolumn{2}{|c|}{ HD 36402} \\
\hline & & & & IVC & HVC & IVC & HVC & IVC & $\mathrm{HVC}$ \\
\hline Ly $R(0), 1-0$ & 1092.194 & $59 \pm 28$ & b (LR1, 1-0) & $<16$ & - & - & - & - & - \\
\hline Ly $R(0), 2-0$ & 1077.138 & $76 \pm 22$ & b (LR1, 2-0) & - & - & $<16$ & - & - & - \\
\hline Ly $R(0), 4-0$ & 1049.366 & $\mathrm{n}, \mathrm{b} ?$ & $<30$ & - & $<60$ & - & $<14$ & - & $<7$ \\
\hline Ly $R(0), 8-0$ & 1001.826 & $79 \pm 17$ & $\mathrm{~b} ?(\mathrm{LR} 1)$ & - & - & $<25$ & $<19$ & $<12$ & $<12$ \\
\hline Ly $R(0), 10-0$ & 981.441 & $77 \pm 23$ & $\mathrm{~b} ?(\mathrm{LR} 1)$ & $<17$ & $<56$ & - & - & $<18$ & $<16$ \\
\hline Ly $R(1), 1-0$ & 1092.732 & $46 \pm 25$ & b (LR0,1-0) & $<26$ & - & - & - & - & - \\
\hline We $Q(1), 1-0$ & 986.798 & b (LR3, 10-0, LMC) & $75 \pm 22$ & - & - & - & - & - & $<26$ \\
\hline Ly $R(1), 2-0$ & 1077.698 & $63 \pm 19$ & b (LR0, 2-0) & $<61$ & - & $<26$ & - & $<13$ & - \\
\hline Ly $\mathrm{P}(1), 5-0$ & 1038.156 & $54 \pm 31$ & $\mathrm{~b}(\mathrm{LR} 0)$ & - & - & $<17$ & - & & - \\
\hline Ly $R(1), 8-0$ & 1002.457 & $71 \pm 22$ & $34 \pm 19$ & $<63$ & $<47$ & $<21$ & $<29$ & $<11$ & - \\
\hline We $Q(2), 0-0$ & 1010.941 & $<38$ & $65 \pm 22$ & - & $<36$ & $<12$ & $<12$ & $<9$ & $<9$ \\
\hline Ly $R(2), 4-0$ & 1051.497 & $\mathrm{n}$ & $54 \pm 23$ & $<30$ & - & $<22$ & - & $<7$ & $<8$ \\
\hline Ly $\mathrm{P}(2), 7-0$ & 1016.472 & $\mathrm{n}$ & $<57$ & $<25$ & $<35$ & $<21$ & $<10$ & $<13$ & $<15$ \\
\hline Ly $\mathrm{P}(2), 11-0$ & 975.343 & $\mathrm{n}$ & $<42$ & - & - & - & - & - & - \\
\hline Ly $\mathrm{P}(3), 3-0$ & 1070.142 & $<36$ & $33 \pm 17$ & $<40$ & - & - & $<23$ & $<16$ & - \\
\hline Ly $R(3), 4-0$ & 1053.976 & $\mathrm{~b} ?$ & $47 \pm 23$ & $<75$ & - & $<14$ & $<20$ & - & $<19$ \\
\hline Ly $\mathrm{P}(3), 4-0$ & 1056.469 & $\mathrm{n}$ & $\mathrm{n}$ & $<18$ & $<18$ & - & - & - & - \\
\hline Ly $\mathrm{P}(3), 5-0$ & 1043.498 & $39 \pm 27$ & $\mathrm{n}$ & - & $<33$ & - & - & $<18$ & - \\
\hline Ly $R(3), 8-0$ & 1006.418 & $\mathrm{n}, \mathrm{b} ?$ & $63 \pm 30$ & - & $<63$ & $<20$ & - & - & $<28$ \\
\hline Ly $R(4), 4-0$ & 1057.379 & - & $46 \pm 23$ & - & - & - & - & - & - \\
\hline Ly $\mathrm{P}(5), 4-0$ & 1065.594 & - & $<31$ & - & - & - & - & - & - \\
\hline Ly $P(6), 3-0$ & 1085.382 & - & $<54$ & - & - & - & - & - & - \\
\hline Ly $R(6), 7-0$ & 1030.064 & - & $65 \pm 25$ & - & - & - & - & - & - \\
\hline
\end{tabular}

Wavelengths are taken from Morton \& Dinerstein (1976). Some equivalent widths have not been measured due to: (n) noise, (f) low flux, (b) blend. Some blends could not be identified.

consistent within the range of errors. The gas on these lines of sight thus should be fully thermalised. For the sight lines towards $\mathrm{Sk}-67166$ and $\mathrm{HD} 36402$ (A) $T_{02}$ is significantly higher than $T_{01}$. In this dilute, well illuminated gas the $J=2$ states presumably are already excited by UV pumping, so that they are overpopulated compared to what would be expected from just collisional excitation. Here we also find high excitation temperatures of the upper states, again due to the low density preventing efficient self shielding. Thus a clear distinction between collisionally excited states and states excited by UV pumping is not possible.

The column densities on all sight lines are quite similar for the upper, UV pumping excited $J$ levels. Only the lower, collisionally excited levels vary significantly in column density. This allows the conclusion that we look in the direction of the LMC through a layer of quite homogeneously distributed UV pumping excited molecular gas, with regions of differing column densities in the cooler lower states.

The $\mathrm{H}_{2}$ absorption component $\mathrm{B}$ towards HD 36402 at $+35 \mathrm{~km} \mathrm{~s}^{-1}$ seems to be compact enough to contain a shielded core at a Boltzmann temperature of about
$T_{\mathrm{ex}}<100 \mathrm{~K}$. Similar conditions are found on the two other high column density sight lines, towards HD 269546 and LH 10:3120. The upper excitation levels indicate a high equivalent temperature of $T_{\mathrm{UV}}>1000 \mathrm{~K}$ due to $\mathrm{UV}$ pumping, occuring probably in the outer cloud regions.

The atomic absorption found near $v_{\text {hel }} \simeq+15 \mathrm{~km} \mathrm{~s}^{-1}$ in the HD 36402 spectrum is rather wide (from -15 to $+45 \mathrm{~km} \mathrm{~s}^{-1}$ ) and is, as noted before, probably a blend of the two velocity components seen in $\mathrm{H}_{2}$ at $v_{\text {hel }} \simeq$ $-5 \mathrm{~km} \mathrm{~s}^{-1}$ and $v_{\text {hel }} \simeq+35 \mathrm{kms}^{-1}$. An exception is the $2852 \AA$ line of $\mathrm{Mg}$ I, a tracer of cold gas. This line is narrow $\left(F W H M \approx 30 \mathrm{~km} \mathrm{~s}^{-1}\right)$ and shows no sub-structure. It belongs to the $+35 \mathrm{~km} \mathrm{~s}^{-1}$ component, as follows immediately from a comparison of the $\mathrm{Mg}$ I absorption profile with that of Mg II $\lambda 2802$. From this we conclude that component $\mathrm{B}$ contains gas which is cooler and denser than that in component $\mathrm{A}$.

\subsection{The IVC gas}

We have detected $\mathrm{H}_{2}$ absorption in the IVC gas $\left(v_{\text {hel }} \approx\right.$ $+56 \mathrm{~km} \mathrm{~s}^{-1}$ ) towards HD 269546. On the other lines of sight we have significant upper limits. The distribution of 
Table 5. Local gas $\mathrm{H}_{2}$ column densities $\log N\left[\mathrm{~cm}^{-2}\right]$ and $\mathrm{H}_{2}$ excitation temperatures towards the LMC stars. $v_{\text {hel }}$ is the heliocentric velocity from the ORFEus data, $b$ is the Doppler parameter of the curves of growth

\begin{tabular}{llllll}
\hline $\mathrm{J}$ & HD 269546 & LH 10:3120 & Sk -67166 & HD 36402 (A) & HD 36402 (B) \\
\hline$v_{\text {hel }}\left[\mathrm{km} \mathrm{s}^{-1}\right]$ & $+5 \pm 4$ & $-16 \pm 6$ & $+7 \pm 5$ & $-4 \pm 5$ & $+35 \pm 4$ \\
\hline$b\left[\mathrm{~km} \mathrm{~s}^{-1}\right]$ & $3-4$ & $4-6$ & $4-6$ & $2-4$ & $4-6$ \\
\hline 0 & $17.10_{-0.25}^{+0.15}$ & $17.00_{-0.90}^{+0.50}$ & $14.55_{-0.30}^{+0.25}$ & $14.70_{-0.55}^{+0.90}$ & $15.85_{-0.45}^{+0.35}$ \\
1 & $16.70_{-0.40}^{+0.35}$ & $16.20_{-0.60}^{+0.60}$ & $14.60_{-0.25}^{+0.25}$ & $14.85_{-0.30}^{+0.35}$ & $15.45_{-0.35}^{+0.30}$ \\
2 & $15.50_{-0.40}^{+0.50}$ & $15.80_{-0.40}^{+0.60}$ & $14.65_{-0.35}^{+0.30}$ & $14.30_{-0.25}^{+0.20}$ & $14.35_{-0.15}^{+0.15}$ \\
3 & $15.20_{-0.40}^{+0.45}$ & $15.40_{-0.50}^{+1.10}$ & $14.70_{-0.35}^{+0.35}$ & $<15.00$ & $14.25_{-0.30}^{+0.15}$ \\
4 & $15.00_{-0.40}^{+0.15}$ & $14.80_{-0.30}^{+0.30}$ & $<13.90$ & - & $15.40_{-0.35}^{+0.30}$ \\
5 & $14.90_{-0.50}^{+0.60}$ & $<14.60$ & $<14.60$ & - & $<14.45$ \\
\hline $\mathrm{H}_{2}(\mathrm{total})$ & $17.26_{-0.19}^{+0.16}$ & $17.10_{-0.53}^{+0.44}$ & $15.34_{-0.10}^{+0.15}$ & $15.38_{-0.10}^{+0.40}$ & $16.12_{-0.20}^{+0.24}$ \\
\hline$T_{01}[\mathrm{~K}]$ & $55_{-16}^{+43}$ & $42_{-16}^{+249}$ & $82_{-29}^{+127}$ & $>37$ & $55_{-17}^{+68}$ \\
$T_{02}[\mathrm{~K}]$ & $96_{-18}^{+47}$ & $117_{-38}^{+438}$ & $>185$ & $200_{-100}^{+430}$ & $101_{-19}^{+37}$ \\
$T_{24}[\mathrm{~K}]$ & $670_{-370}^{+1800}$ & $400_{-170}^{+500}$ & $<780$ & - & - \\
$T_{35}[\mathrm{~K}]$ & $>450$ & $\lesssim 650$ & $\lesssim 2200$ & - & $\approx 4400$ \\
\hline
\end{tabular}

Table 6. Halo gas $\mathrm{H}_{2}$ column densities $\log N\left[\mathrm{~cm}^{-2}\right]$ and $\mathrm{H}_{2}$ excitation temperatures towards the LMC stars. For the three rightmost sight lines the first value gives the column density for a Doppler parameter $b=1 \mathrm{~km} \mathrm{~s}^{-1}$, the second assumes the equivalent width at the Doppler limit of the curve of growth, which is quite appropriate for $b$-values larger than about $5 \mathrm{~km} \mathrm{~s}^{-1}$

\begin{tabular}{lllllllll}
\hline $\mathrm{J}$ & \multicolumn{2}{l}{ HD 269546 } & \multicolumn{2}{l}{ LH 10:3120 } & \multicolumn{2}{l}{ Sk -67166} & & \multicolumn{2}{l}{ HD 36402 } \\
& IVC & HVC & IVC & HVC & IVC & HVC & IVC & HVC \\
\hline$v_{\text {hel }}\left[\mathrm{km} \mathrm{s}^{-1}\right]$ & +60 & +120 & +60 & +120 & +60 & +120 & +60 & +120 \\
\hline 0 & $15.30_{-0.40}^{+0.50}$ & $<14.20$ & $<15.6 / 14.0$ & $<18.4 / 14.5$ & $<15.3 / 14.1$ & $<14.6 / 13.8$ & $<14.4 / 13.7$ & $<13.6 / 13.5$ \\
1 & $15.20_{-0.35}^{+0.40}$ & $14.60_{-0.20}^{+0.20}$ & $<18.3 / 14.7$ & $<18.2 / 14.6$ & $<16.0 / 14.1$ & $<17.6 / 14.3$ & $<14.5 / 13.9$ & $<16.6 / 14.0$ \\
2 & $<14.40$ & $14.80_{-0.20}^{+0.20}$ & $<17.3 / 14.4$ & $<17.5 / 14.3$ & $<14.4 / 13.8$ & $<13.9 / 13.6$ & $<13.9 / 13.6$ & $<13.9 / 13.6$ \\
3 & $14.80_{-0.70}^{+0.05}$ & $14.80_{-0.20}^{+0.20}$ & $<16.0 / 14.3$ & $<16.1 / 14.3$ & $<14.8 / 14.0$ & $<16.4 / 14.2$ & $<15.7 / 14.3$ & $<16.3 / 14.2$ \\
4 & - & $14.70_{-0.40}^{+0.40}$ & - & - & - & - & - & - \\
5 & - & $<14.70$ & - & - & - & - & - & - \\
6 & - & $<14.90$ & - & - & - & - & - & - \\
\hline $\mathrm{H}_{2}($ total $)$ & $15.65_{-0.19}^{+0.32}$ & $15.56_{-0.06}^{+0.10}$ & $<18.3 / 15.0$ & $<18.6 / 15.0$ & $<16.1 / 14.6$ & $<17.6 / 14.7$ & $<15.8 / 14.6$ & $<16.8 / 14.5$ \\
\hline$T_{\text {ex }}[\mathrm{K}]$ & $<150$ & - & - & - & - & - & - & - \\
$T_{\mathrm{UV}}[\mathrm{K}]$ & - & $>1000$ & - & - & - & - & - \\
\hline
\end{tabular}

the column densities over the various rotationally excited levels of $\mathrm{H}_{2}$ (see Table 6) can be used to calculate the kinetic temperature of the gas. Using the two para states $J=0$ and 2 we find an upper limit of $T_{02}<150 \mathrm{~K}$. Level $J=3$ has been detected in absorption, too, but the uncertainty in the column density is large. Still, its detection suggests that the UV photon field is quite important in the IV gas. In fact the full continuum flux from the Milky Way disk is available for photo-excitation, unattenuated by $\mathrm{H}_{2}$ line absorption (see Appendix).

Intermediate velocity gas is found in many high latitude directions in the Milky Way (see Danly 1992). As noted in the introduction, the present detection of $\mathrm{H}_{2}$ absorption in IVC gas is the third case of $\mathrm{H}_{2}$ proven to be existent in such gas.

On our four lines of sight IVC absorptions in the metals are seen at $v_{\text {hel }}=50$ to $70 \mathrm{~km} \mathrm{~s}^{-1}$ (see Table 6 ). These absorptions are weaker than those by disk and HVC gas.
They are often poorly resolved from these other velocity components, so the uncertainties in the IVC equivalent widths are large. Metal column densities appear to be large with respect to H I (see Table 7), except for oxygen. This abundance pattern is also seen in the HVC gas (see next section).

The relative absence of $\mathrm{O}$ (see also Table 8) can be interpreted as due to partial photoionization of the gas, affecting predominantly $\mathrm{H}$ and $\mathrm{O}$, and less the other elements, because the ionization potential of $\mathrm{HI}$ and $\mathrm{O} \mathrm{I}$ is $13.6 \mathrm{eV}$, lower than that of Si II $(16.3 \mathrm{eV})$, S II $(23.33 \mathrm{eV})$ or Fe II $(16.2 \mathrm{eV})$. O II does not have absorption lines in the UV. Therefore we estimate the $N(\mathrm{O}$ II $)$ to $N(\mathrm{O}$ I) ratio by comparing the $\mathrm{O}$ I and $\mathrm{S}$ II column densities. Here we use the fact that the oxygen and sulfur abundance ratio is only weakly related to the metallicity of the gas (see for example Samland 1998) as well as that the oxygen and sulfur abundances are barely affected by deposition onto dust 
Table 7. Column densities of metals and $\mathrm{HI}$ as $\log N\left[\mathrm{~cm}^{-2}\right]$ towards the four program stars. $v_{\text {hel }}\left[\mathrm{km} \mathrm{s}^{-1}\right]$ is the heliocentric radial velocity of the absorption components from the ORFEus data, $b\left[\mathrm{~km} \mathrm{~s}^{-1}\right]$ is the Doppler parameter of the curves of growth

\begin{tabular}{|c|c|c|c|c|c|c|c|c|c|c|c|c|}
\hline & \multicolumn{3}{|l|}{ HD 269546} & \multicolumn{3}{|c|}{ LH 103120} & \multicolumn{3}{|l|}{$\mathrm{Sk}-67166$} & \multicolumn{3}{|l|}{ HD 36402} \\
\hline & disk & IVC & $\mathrm{HVC}$ & disk & IVC & $\mathrm{HVC}$ & disk & IVC & $\mathrm{HVC}$ & disk & IVC & $\mathrm{HVC}$ \\
\hline$v_{\text {hel }}$ & $-9_{-3}^{+3}$ & $+56_{-7}^{+7}$ & $+118_{-4}^{+4}$ & $-16_{-2}^{+2}$ & $+51_{-5}^{+5}$ & $+122_{-10}^{+10}$ & $-5_{-3}^{+3}$ & $+61_{-3}^{+3}$ & $+125_{-5}^{+5}$ & $+15_{-4}^{+4}$ & $+70_{-4}^{+4}$ & $+131_{-3}^{+3}$ \\
\hline$b$ & $22_{-3}^{+3}$ & $7_{-3}^{+3}$ & $11_{-3}^{+3}$ & - & - & - & $11-19$ & $5_{-2}^{+2}$ & $22_{-3}^{+3}$ & $15_{-5}^{+5}$ & $9_{-2}^{+2}$ & $18_{-6}^{+7}$ \\
\hline $\mathrm{OI}_{\mathrm{I}}$ & $16.35_{-0.3}^{+0.3}$ & $14.3_{-0.9}^{+0.5}$ & $15.2_{-0.2}^{+0.3}$ & $>15.8$ & - & $>15.0$ & $16.2_{-0.5}^{+0.3}$ & $15.0_{-0.7}^{+1.2}$ & $14.85_{-0.2}^{+0.2}$ & $16.2_{-0.3}^{+0.3}$ & $15.25_{-0.3}^{+0.3}$ & $15.2_{-0.2}^{+0.3}$ \\
\hline Si II & $15.4_{-0.15}^{+0.15}$ & $14.6_{-0.5}^{+0.5}$ & $15.1_{-0.2}^{+0.3}$ & $>13.9$ & $>13.5$ & $>13.6$ & $15.35_{-0.10}^{+0.15}$ & $14.0_{-0.7}^{+0.5}$ & $14.3_{-0.2}^{+0.3}$ & $15.35_{-0.15}^{+0.15}$ & $14.0_{-0.5}^{+0.5}$ & $14.6_{-0.3}^{+0.3}$ \\
\hline Fe II & $15.0_{-0.2}^{+0.2}$ & $14.5_{-0.3}^{+0.3}$ & $14.4_{-0.3}^{+0.3}$ & $>15.1$ & $>13.5$ & $>14.2$ & $14.85_{-0.5}^{+0.2}$ & $14.4_{-0.6}^{+0.4}$ & $14.1_{-0.3}^{+0.4}$ & $14.5_{-0.2}^{+0.2}$ & $14.2_{-0.4}^{+0.5}$ & $14.2_{-0.4}^{+0.3}$ \\
\hline S II & $15.2_{-0.2}^{+0.2}$ & $14.2_{-0.2}^{+0.3}$ & $14.65_{-0.3}^{+0.3}$ & $>15.0$ & $>15.0$ & $>15.1$ & $15.3_{-0.2}^{+0.2 .5}$ & $14.8_{-1.5}^{+1.5}$ & $14.85_{-0.2}^{+0.2}$ & $15.1_{-0.2}^{+0.2}$ & $14.3_{-0.2}^{+0.4}$ & $14.7_{-0.2}^{+0.4}$ \\
\hline Mg I & $12.6_{-0.2}^{+0.3}$ & $12.6_{-0.3}^{+1.4}$ & $12.8_{-0.4}^{+0.7}$ & - & - & - & $12.4_{-0.2}^{+0.2}$ & $11.9_{-0.4}^{+0.6}$ & $12.25_{-0.2}^{+0.2}$ & $12.6_{-0.3}^{+0.5}$ & $12.3_{-0.3}^{+0.5}$ & $12.3_{-0.3}^{+0.4}$ \\
\hline Mg II & $15.45_{-0.6}^{+0.7}$ & $12.85_{-0.9}^{+0.8}$ & - & - & - & - & $14.5_{-0.6}^{+1.0}$ & $14.3_{-0.8}^{+1.4^{+}}$ & $13.8_{-0.5}^{+1.2}$ & $15.6_{-1.2}^{+0.9}$ & $13.5_{-0.5}^{+0.9}$ & $14.2_{-0.5}^{+0.8}$ \\
\hline $\mathrm{H} \mathrm{I}$ & $20.54_{-0.08}^{+0.07}$ & $18.2_{w}$ & $19.05_{w}$ & $20.7_{-8}^{+c}$ & $\begin{array}{l}8 \\
8^{r}\end{array}$ & & $20.54_{-0.09}^{+0.08}$ & $<18.2_{p}$ & $<18.0_{p}$ & $20.73_{s}$ & $18.64_{w}$ & $18.64_{w}$ \\
\hline
\end{tabular}

$w$ from Wierig \& de Boer (1998) based on Parkes 15’ beam $21 \mathrm{~cm}$ data by McGee et al. (1983) and McGee \& Newton (1986) $r$ from Richter (2000a).

$p$ from a Parkes $15^{\prime}$ beam $21 \mathrm{~cm}$ emission profile (centered $\approx 14^{\prime}$ off the position of Sk -67166$)$ by McGee \& Newton $(1986)$. $s$ from Savage \& de Boer (1981).

Note that the O I column densities in disk gas have to be treated as lower limits (see Sect. 4.1).

grains. In the neutral interstellar medium the common value of $\log [N(\mathrm{O}) / N(\mathrm{~S})]$ is $\approx 1.2 \pm 0.2$ (for the $\mathrm{O}$ I abundance in the neutral ISM see Meyer et al. 1998). In contrast, towards HD 269546, we find $\log [N(\mathrm{O} \mathrm{I}) / N(\mathrm{~S} \mathrm{II})]=$ $0.1_{-1.0}^{+0.5}$. Thus the actual oxygen column density is presumably higher by 1.1 dex than that of O I. For temperatures above $\approx 1000 \mathrm{~K}$ the ionization level of oxygen is coupled to that of hydrogen due to charge exchange reactions (see e.g. Field \& Steigman 1971). Because of $N(\mathrm{H}$ II $) / N(\mathrm{H} \mathrm{I}) \approx \frac{9}{8} N(\mathrm{O}$ II $) / N(\mathrm{O}$ I $)$, the hydrogen column density has to be increased by the same amount as the oxygen column density, which would mean an ionization level of more than $90 \%$ if our assumptions are correct. The line of sight to HD 36402 is different, it has rather normal abundances and $N(\mathrm{O} \mathrm{I}) / N(\mathrm{~S} \mathrm{II})$ indicates no ionization.

On most sight lines (except towards HD 269546) noise and blending only allow to derive upper limits for the $\mathrm{H}_{2}$ content in the IVC gas (see Table 6). To obtain these we fitted the upper limits for the equivalent widths to curves of growth with a $b$-value of $1 \mathrm{kms}^{-1}$ and to the Doppler limit of the curve of growth. The lower $b$-value should give a distinct limit in the case of very cold gas cores. The Doppler limit largely applies for all $b$-values $\geq$ $5 \mathrm{~km} \mathrm{~s}^{-1}$. Experience shows that in gas this dilute $b$-values are normally significantly larger than $1 \mathrm{~km} \mathrm{~s}^{-1}$, similar to metal $b$-values.

The metal and $\mathrm{H}_{2}$ absorptions together indicate that the fraction of $\mathrm{H}_{2}$ of all $\mathrm{H}$ is rather small, near $10^{-3.5}$, when including the correction for fractional ionization of $\mathrm{H}$ of $90 \%$ as estimated above. These lines of sight sample IV gas which has dilute and ionized portions as well as cooler and denser portions.

Wolfire et al. (1995) investigated the pressure range for which stable two-phase gas can exist in the galactic halo.
Table 8. $\log [\mathrm{X} / \mathrm{HI}]-\log [\mathrm{X} / \mathrm{H}]_{\odot}$ in disk, IVC, and HVC gas. Solar abundances are taken from Savage \& Sembach (1996). The uncertainties are at least as large as those of the metal column densities. The overabundances of some elements relative to $\mathrm{HI}$ and $\mathrm{O}$ I indicate a significant degree of ionization in the HVC and the IVC. The lower limits for oxygen in disk gas reflect the uncertainties due to geocoronal $\mathrm{O}$ I emission at the velocity of the disk component

\begin{tabular}{llccccc}
\hline & & $\mathrm{O}$ & $\mathrm{S}$ & $\mathrm{Si}$ & $\mathrm{Fe}$ & $\mathrm{Mg}$ \\
\hline \multirow{4}{*}{ disk } & HD 269546 & $\geq-1.2$ & -0.5 & -0.6 & -1.0 & -0.7 \\
& Sk -67 166 & $\geq-1.2$ & -0.5 & -0.7 & -1.3 & -1.6 \\
& HD 36402 & $\geq-1.4$ & -0.8 & -0.9 & -1.7 & -0.7 \\
\hline \multirow{2}{*}{ IVC } & HD 269546 & -0.8 & +0.8 & +1.0 & +0.8 & +0.8 \\
& HD 36402 & -0.3 & +0.5 & -0.1 & +0.1 & -0.7 \\
\hline \multirow{2}{*}{ HVC } & HD 269546 & -0.7 & +0.4 & +0.5 & -0.2 & - \\
& HD 36402 & -0.3 & +0.9 & +0.5 & +0.1 & -0.0 \\
\hline
\end{tabular}

For cloud heights between $1 \mathrm{kpc}$ and $3 \mathrm{kpc}$ their models give $P / k$ values of $10^{3.2} \mathrm{~K} \mathrm{~cm}^{-3}$ to $10^{3.7} \mathrm{~K} \mathrm{~cm}^{-3}$. Under the assumption of a pressure of about $10^{3.5} \mathrm{~K} \mathrm{~cm}^{-3}$ and with the upper limit for the kinetic temperature of $\mathrm{H}_{2}$, it is possible to estimate as lower limit $n_{\mathrm{H}} \gtrsim 20 \mathrm{~cm}^{-3}$ for the particle density in the molecular core of the IVC. The H I column density divided by this number density yields a size of $\approx 0.025 \mathrm{pc}$. This is an upper limit for the cold core of the cloud. It is at the same time a lower limit for the total cloud since the outer regions are certainly warmer than $150 \mathrm{~K}$. 

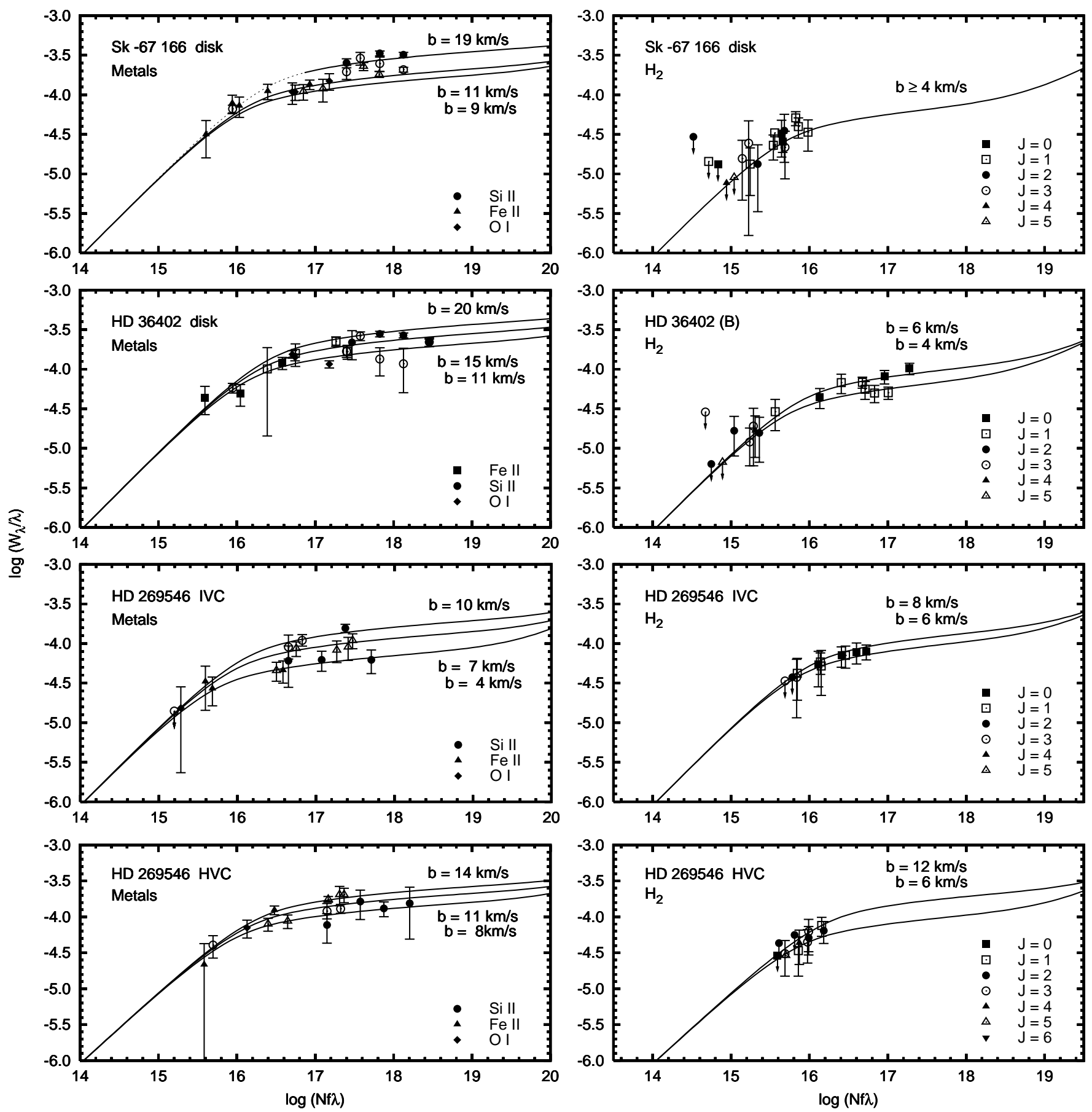

Fig. 3. Examples for the curves of growth for absorption by metals (left) and $\mathrm{H}_{2}$ (right) are shown. For the metals, filled symbols represent ORFEUS data, open symbols those from the IUE. The drawn curves represent single cloud absorption with indicated $b$-value

\subsection{The halo HVC gas}

The HVC gas on the LMC line of sight was known (as was the IVC gas) since the first IUE observations (Savage \& de Boer 1979). Absorption by $\mathrm{H}_{2}$ was detected in ORFEUS spectra towards HD 269546 and essentials have been presented by Richter et al. (1999a). Here we will discuss also aspects which could not be covered in that paper.
The distance of the HVC is still unknown. However, HVCs have probably typical distances of $z \gtrsim 3 \mathrm{kpc}$ (Kaelble et al. 1985; van Woerden et al. 1999; see also review by Wakker \& van Woerden 1997). Taking that distance in $z$ implies that the ORFEus lines of sight intersect the $+120 \mathrm{~km} \mathrm{~s}^{-1} \mathrm{HVC}$ over a projected width of $\gtrsim 350 \mathrm{pc}$. The three sight-lines for which metal column densities can be given correspond to a width of $\gtrsim 130 \mathrm{pc}$ in the assumed distance. Though the Doppler parameters have values 


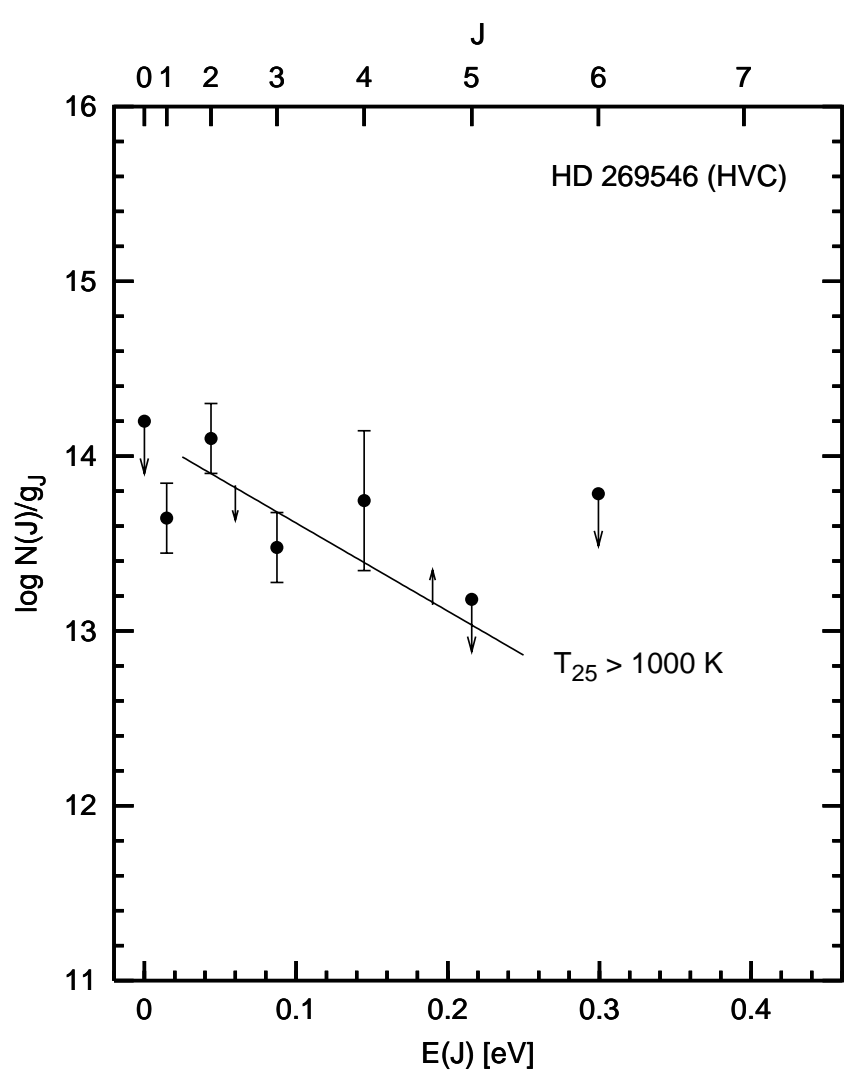

Fig. 4. Excitation plot for the $\mathrm{H}_{2} \mathrm{HVC}$ component towards HD 269546. The alternation in column densities is due to the ortho-to-para ratio of $\simeq 1$. The excitation temperature due to UV pumping is derived to be $>1000 \mathrm{~K}$

between 11 and $22 \mathrm{kms}^{-1}$, the column densities are similar towards the different targets.

On all lines of sight $\mathrm{O} I$ is depleted relative to the other metals. Applying the ionization correction described in the previous section, we find $\log N(\mathrm{HII}) / N(\mathrm{HI}) \approx$ $\log N(\mathrm{O}$ II $) / N(\mathrm{O}$ I $)$ as $0.7,1.2$, and 0.7 dex towards HD 269546, Sk -67166, and HD 36402 respectively. This means that in the HV gas roughly only $5-20 \%$ of the hydrogen is neutral.

The column densities of metals are given in Table 7 , the apparent depletions (without ionization correction) in Table 8. Taking $\mathrm{S}$ as the reference for the abundances we find that $\mathrm{Si}$ and $\mathrm{Fe}$ are depleted by factors ranging from 2 to 4 . Based on the Parkes H I column densities and the ionization correction, the abundances of $\mathrm{S}$, Si, and Fe turn out to be similar to those in the warm local ISM. Of course there are considerable uncertainties in the ionization correction as well as in the $\mathrm{H}$ I column densities (because of the Parkes beam size). Given the metal content neither a galactic nor an LMC origin of this HVC can be excluded.

With the $\mathrm{Mg}$ I to $\mathrm{Mg}$ II ratio it is possible to estimate temperature, density, and extent of the HVC. We assume a $z$ height between $3 \mathrm{kpc}$ and $10 \mathrm{kpc}$ and a pressure $\log (P / k) \approx 3.3$ (a value from the models of Wolfire et al. 1995). If the gas is in ionization equilibrium and if charge exchange reactions are neglegible, $N(\mathrm{Mg} \mathrm{II}) / N(\mathrm{Mg} \mathrm{I})$ is

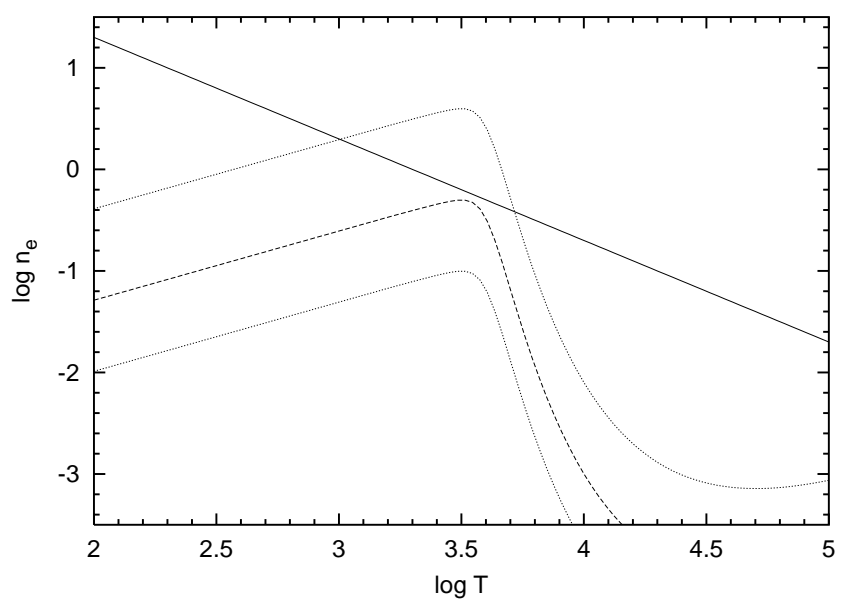

Fig. 5. For the HVC on the line of sight to HD 36402 we show $n_{\mathrm{e}}(T)=\frac{\Gamma}{\alpha(T)} \cdot \frac{N(\mathrm{Mg} \text { I })}{N(\mathrm{Mg} \mathrm{II})}$ (dashed curve, with dotted curves representing the errorbars of $\left.\frac{N(\mathrm{Mg} \text { I })}{N(\mathrm{Mg} \text { II })}\right)$. The straight line gives $n_{\mathrm{e}}=\frac{P_{\mathrm{e}}}{k} \cdot \frac{1}{T}$ for the pressure $n_{\mathrm{e}} T=10^{3.3} \mathrm{~cm}^{-3} \mathrm{~K}$, as indicated by the models of Wolfire et al. (1995) for reasonable $z$ heights. The intersection allows an estimate of density and temperature in the $\mathrm{HVC}$

determined by $\frac{\Gamma}{\alpha n_{\mathrm{e}}}$, with the photoionization rate $\Gamma$, the temperature dependent recombination coefficient $\alpha$, and the electron density $n_{\mathrm{e}}$. Fits for the different contributions to $\alpha$ can be found in Péquignot \& Aldrovandi (1986), Shull \& Van Steenberg (1982) and Nussbaumer \& Storey (1986). The flux of ionizing photons and the resulting photoionization rates at the location of the HVC are unknown. We use the photoionization rate for $\mathrm{Mg}$ I in the galactic disk given in de Boer et al. (1973), scaled down by a factor of $\approx 3$ to the halo radiation field as estimated by Bregman \& Harrington (1986). In Fig. 5 we plot $n_{\mathrm{e}}(T)=\frac{\Gamma}{\alpha(T)} \cdot \frac{N(\mathrm{Mg} \mathrm{I})}{N(\mathrm{Mg} \text { II })}$ and $n_{\mathrm{e}}=\frac{P_{\mathrm{e}}}{k} \cdot \frac{1}{T}$ for the HVC gas on the HD 36402 line of sight. The intersection of these curves provides information about temperature and electron density in the cloud. It indicates $0.08<n_{\mathrm{e}}<3 \mathrm{~cm}^{-3}$ at temperatures $1000<T<6000 \mathrm{~K}$. Because of the high degree of ionization the dominant source of electrons is hydrogen and $n_{\mathrm{e}}$ equals $n\left(\mathrm{H}^{+}\right) \approx 5 \cdot n(\mathrm{HI})$. With the value for the column density $N(\mathrm{HI})=4.410^{18} \mathrm{~cm}^{-2}$, we now can estimate the extent of the cloud along the line of sight to be of the order of $1-100 \mathrm{pc}$.

In the $\mathrm{HVC}$ gas $\mathrm{H}_{2}$ is only found towards HD 269546 (Richter et al. 1999a). Here the $b$-value for $\mathrm{H}_{2}$ is $9 \mathrm{~km} \mathrm{~s}^{-1}$, only $2 \mathrm{~km} \mathrm{~s}^{-1}$ lower than that for the metals. As with the IVC, we can assume that the full disk radiation field operates on HVC gas, too (see Appendix). The excitation temperature ( $\geq 1000 \mathrm{~K}$, see Fig. 4$)$ and the ortho-to-para ratio $(\approx 1)$ of $\mathrm{H}_{2}$ point to deviations from thermodynamic equilibrium conditions in this part of the HVC. This can be explained by quite different mechanisms, such as $\mathrm{H}_{2}$ formation or a former cloud core, which is now exposed to and photodissociated by UV flux from the galactic disk. Spitzer \& Zweibel (1974) calculated the steady state $\mathrm{H}_{2}$ photo excitation for different radiation fields, densities, 
and temperatures. It turns out that none of their models yields excitation temperatures as high as measured in the $\mathrm{HVC}$, except for the case of recent $\mathrm{H}_{2}$ formation. Though it is conceivable that in the galactic fountain model $\mathrm{H}_{2}$ is being formed in the cooling gas falling back to the galactic plane, the present data do not allow a full assessment of the physical conditions in this cloud.

As for the IVC, also for the HVC only upper limits are available for $\mathrm{H}_{2}$ towards the remaining targets (Table 6), again derived assuming $b$-values of $1 \mathrm{~km} \mathrm{~s}^{-1}$ and $b>5 \mathrm{~km} \mathrm{~s}^{-1}$. In most cases the deviation between the upper limits of para- $\mathrm{H}_{2}$ states and their corresponding ortho-states is much larger than one would expect for typical $\mathrm{H}_{2}$ excitation. Thus, if ortho- and para-states are both taken into account, the upper limits for many of the excitational states are smaller than stated in Table 6 .

\section{Overall conclusions}

It has been suggested that HVCs are building blocks of the Local Group containing low metallicity gas (e.g. Blitz et al. 1999). The IVC and the HVC in front of the LMC do not fit in this model. Metal abundances and the existence of $\mathrm{H}_{2}$ support the galactic fountain model for this IVC/HVC complex. Though the underabundance of metals in the HVC seems superficially consistent with a Magellanic Cloud origin of that gas, the presence of $\mathrm{H}_{2}$ points to the existence of dust and thus intrinsically higher metallicity.

Acknowledgements. We thank the ORFEus team for making and operating ORFEUs and providing us with the data. HB is supported by the GK The Magellanic Clouds and other dwarf galaxies, PR was supported by grant 50 QV 9701-3 from the DARA (now DLR), OM by grant Bo 779/24 from the Deutsche Forschungsgemeinschaft (DFG). Part of the data has been collected under the DARA ORFEUs guest observer programme. We also made use of the public archive of IUE spectra.

\section{Appendix: Radiation field in the halo and $\mathrm{H}_{2}$ photo excitation}

In order to interpret the level of photo excitation of $\mathrm{H}_{2}$ in the halo clouds one has to know the radiation field. In the lower halo the radiation density is essentially the same as that in the Milky Way (MW) disk. Very far out the radiation field is diluted because the gas will not see so large a solid angle of Milky Way radiation.

What matters for $\mathrm{H}_{2}$ and its photo excitation is the amount of photons at the wavelength of the $\mathrm{H}_{2}$ absorption lines. Nominally, these photons have been absorbed in the molecular gas of the MW disk. A halo cloud will, however, see radiation from different parts of the Milky Way disk having different radial velocities due to the galactic rotation. Moreover, halo clouds will normally not have zero velocity with respect to the MW disk.

We have performed a simple integration over the galactic disk to determine the distribution of the velocity shifts.
A map of the MW with model radial velocities was divided in $20^{\circ}$ sectors overlain with circles with steps of $1 \mathrm{kpc}$ in radius. For each of these curved boxes the average LSR radial velocity was estimated by eye. All these values, weighted with the surface area of the box and with the distance, lead to a histogram of distance related velocity shifts. From the histogram it is immediately clear, that $>75 \%$ of the radiation from the disk arrives in the halo with a shift of less than $30 \mathrm{~km} \mathrm{~s}^{-1}$. Halo clouds with a velocity near $0 \mathrm{~km} \mathrm{~s}^{-1}$ will thus recieve $<25 \%$ of the continuum flux level for photo excitation. However, since the great majority of clouds detected in the halo, IVCs and HVCs alike, have a radial velocity differing by more than $30 \mathrm{~km} \mathrm{~s}^{-1}$ from the LSR, the $\mathrm{H}_{2}$ in these halo clouds sees almost the full continuum radiation at the wavelengths of the $\mathrm{H}_{2}$ absorption lines, i.e., unattenuated by the $\mathrm{H}_{2}$ line disk absorption. Almost the full continuum flux is available for photo excitation.

\section{References}

Barnstedt, J., Kappelmann, N., Appenzeller, I., et al. 1999, A\&AS, 134, 561

Blitz, L., Spergel, D. N., Teuben, P. J., et al. 1999, ApJ, 514,818

Bregman, J. N., \& Harrington, J. P. 1986, ApJ, 309, 833

Chu, Y.-H., Wakker, B., Mac Low, M.-M., \& García-Segura, G. 1994, AJ, 108, 1696

Danly, L., Lockman, F. J., Meade, M. R., \& Savage, B. D. 1992, ApJS, 81, 125

de Boer, K. S., Koppenaal, K., \& Pottasch, S. R. 1973, A\&A, 28,145

de Boer, K. S., \& Nash, A. G. 1982, ApJ, 255, 447

de Boer, K. S., Fitzpatrick, E. L., \& Savage, B. D. 1985, MNRAS, 217, 115

de Boer, K. S., Grewing, M., Richtler, T., et al. 1987, A\&A, 177, L37

de Boer, K. S., Morras, R., \& Bajaja, E. 1990, A\&A, 233, 523

de Boer, K. S., Richter, P., Bomans, D. J., Heithausen, A., \& Koornneef, J. 1998, A\&A, 338, L5

Field, G. B., \& Steigman, G. 1971, ApJ, 166, 59

Grewing, M., \& Schulz-Lüpertz, E. 1981, Mitt. Astron. Ges., 52,79

Gringel, W., Barnstedt, J., de Boer, K. S., et al. 2000, A\&A, 358, L37

Jenkins, E. B., Drake, J. F., Morton, D. C., et al. 1973, ApJ, 181, L122

Kaelble, A., de Boer, K. S., \& Grewing, M. 1985, A\&A, 143,408

Lockman, F. J., Hobbs, L. M., \& Shull, J. M. 1986, ApJ, 301,380

McGee, R. X., \& Newton, L. M. 1986, Proc. Austral. Astron. Soc., 6, 358

McGee, R. X., Newton, L. M., \& Morton, D. C. 1983, MNRAS, 205, 1191

Meyer, D. M., Jura, M., \& Cardelli, J. A. 1998, ApJ, 493, 222

Morton, D. C. 1991, ApJS, 77, 119

Morton, D. C., \& Dinerstein, H. L. 1976, ApJ, 204, 1

Nussbaumer, H., \& Storey, P. 1986, A\&AS, 64, 545

Parker, J. W., Garmany, C. D., Massey, P., \& Walborn, N. R. 1992, AJ, 103, 1205 
Péquignot, D., \& Aldrovandi, S. M. V. 1986, A\&A, 161, 169

Richter, P., de Boer, K. S., Widmann, H., et al. 1999a, Nature, 402, 386

Richter, P., de Boer, K. S., Bomans, D. J., et al. 1999b, A\&A, 351,323

Richter, P. 2000a, A\&A, 359, 1111

Richter, P., Savage, B. D., Wakker, B. P., et al. 2000b, ApJ, accepted

Samland, M. 1998, ApJ, 496, 155

Savage, B. D., Drake, J. F., Budich, W., \& Bohlin, R. C. 1977, ApJ, 216, 291

Savage, B. D., \& de Boer, K. S. 1979, ApJ, 230, L77

Savage, B. D., \& de Boer, K. S. 1981, ApJ, 243, 460

Savage, B. D., \& Sembach, K. R. 1996, ARA\&A, 34, 279

Savage, B. D., Jenkins, E. B., Joseph, C. L., \& de Boer, K. S. 1989, ApJ, 345, 393
Shull, J. M., \& Van Steenberg, M. 1982, ApJS, 48, 95

Spitzer, L., \& Zweibel, E. G. 1974, ApJ, 191, 127

Vacca, W. D., \& Torres-Dodgen, A. V. 1990, ApJS, 73, 685

van Woerden, H., Schwarz, U. J., Peletier, R. F., Wakker, B. P., \& Kalberla, P. M. W. 1999, Nature, 400, 138

Wakker, B. P., \& van Woerden, H. 1997, ARA\&A, 35, 217

Wayte, S. R. 1990, ApJ, 355, 473

Widmann, H., de Boer, K. S., Richter, P., et al. 1998, A\&A, 338, L1

Wierig, B., \& de Boer, K. S. 1998, in The Magellanic Clouds and other dwarf galaxies, ed. T. Richtler, \& J. Braun (Shaker Verlag, Aachen), 305

Wilcots, E. M., Hodge, P. W., \& King, N. 1996, ApJ, 458, 580

Wolfire, M. G., McKee, C. F., Hollenbach, D., \& Tielens, A. G. G. M. 1995, ApJ, 453, 673

Wright, E. L., \& Morton, D. C. 1979, ApJ, 227, 483 